\title{
Factors Affecting the Swallowing Dysfunction Following Oral Cancer Surgery
}

\author{
Narihiro Kodama, $\mathrm{PhD}^{1}$, Yoshihiko Kumai, $\mathrm{MD}, \mathrm{PhD}^{2}$, Takumi Miyamoto, $\mathrm{BS}^{3}$, Keigo Matsubara, $\mathrm{PhD}^{1}$, \\ Yasuhiro Samejima, $\mathrm{MD}, \mathrm{PhD}^{4}$, Yorihisa Orita, $\mathrm{MD}, \mathrm{PhD}^{3}$ \\ ${ }^{1}$ Department of Rehabilitation, Kumamoto Health Science University, Kumamoto; \\ ${ }^{2}$ Department of Otolaryngology Head and Neck Surgery, Nagasaki University Graduate School of Medicine, Nagasaki; \\ ${ }^{3}$ Department of Otolaryngology Head and Neck Surgery, Kumamoto University Graduate School of Medicine, Kumamoto; \\ ${ }^{4}$ Department of Otolaryngology, Kumamoto Kinoh Hospital, Kumamoto, Japan
}

Objective To investigate the factors affecting the postoperative swallowing dysfunction in patients who underwent oral cancer surgery.

Methods Retrospective review of clinical records of 70 patients (50 males and 20 females) who underwent oral cancer surgeries from July 2007 to April 2015 were enrolled. Multiple regression analysis was performed using the Food Intake LEVEL Scale (FILS) at discharge as the objective variable and age, tumor size, resection of the tongue base, suprahyoid muscle resection, segmental mandibulectomy, neck dissection and radiation therapy as the explanatory variables in 70 patients. In addition, multiple regression analysis was performed between objective variables, which include maximum hyoid bone movement, laryngeal elevation delay time, pharyngeal constriction ratio (PCR), residue in the vallecular and pear-shaped depression (pyriform sinuses), and Penetration-Aspiration Scale score and one of the main factors representing the characteristics of each case as the explanatory variables, and age was treated as an adjustment factor in 23 patients.

Results The FILS shows significant negative correlation by age and resection of the tongue base. In videofluoroscopic swallowing study, the maximum movement, PCR and residue in the vallecular are significantly correlated with factors demonstrating the characteristic for each case.

Conclusion It was suggested that in elderly patients, the presence of more than half of the tongue base resection, suprahyoid muscle resection and neck dissection cause severe dysphagia after surgery.

Keywords Oral cancer, Swallowing dysfunction, Food Intake LEVEL Scale (FILS), Videofluoroscopic analysis

Received February 18, 2021; Revised June 8, 2021; Accepted July 5, 2021; Published online October 31, 2021

Corresponding author: Narihiro Kodama

Department of Rehabilitation Kumamoto Health Science University, 325 Izumimachi, Kita-ku, Kumamoto 861-5598, Japan. Tel: +81-96-275-2219, Fax: +81-96-275-2243, E-mail: kodama@kumamoto-hsu.ac.jp

ORCID: Narihiro Kodama (https://orcid.org/0000-0002-5239-3067); Yoshihiko Kumai (https://orcid.org/0000-0002-2808-4539); Takumi Miyamoto (https://orcid.org/0000-0002-9809-3107); Keigo Matsubara (https://orcid.org/0000-0002-6832-9254); Yasuhiro Samejima (https://orcid.org/00000002-4026-6415); Yorihisa Orita (https://orcid.org/0000-0002-2803-9623).

(c) This is an open-access article distributed under the terms of the Creative Commons Attribution Non-Commercial License (http://creativecommons.org/ licenses/by-nc/4.0) which permits unrestricted noncommercial use, distribution, and reproduction in any medium, provided the original work is properly cited. Copyright ( 2021 by Korean Academy of Rehabilitation Medicine 


\section{INTRODUCTION}

Oral cancer often affects vital functions such as breathing, speaking, and swallowing. Patients who undergo surgical treatment for oral cancer tend to incur a swallowing dysfunction. The oral preparatory stage in the swallowing movement is affected by the resection of the oral tongue, which results in chewing dysfunction and influx to the pharynx, and can additionally affect the oral phase of swallowing that results in piecemeal deglutition. The pharyngeal phase is affected by the resection of the tongue base and suprahyoid muscle, which results in pharyngeal residues, reduced laryngeal elevation, and penetration or aspiration. The swallowing dysfunction in these patients depends on several factors. Fujimoto et al. [1] reported that the main factors determining the quality of diet were age, percentage of tongue base resection, and method of postoperative reconstruction. Matsunaga et al. [2] found that patients who underwent excision of more than $50 \%$ of the tongue base required strict dietary limitations. The study by Pauloski et al. [3] indicated that patients who received both surgical intervention and postoperative radiation therapy had significantly reduced oral and pharyngeal swallowing performance compared with patients who received surgery alone.

Conversely, the videographic study by Pauloski et al. [4] using biomechanical analysis to clarify the pathology of dysphagia after surgery for oral cancer revealed that tongue base, pharyngeal wall, hyoid bone, laryngeal, and cricopharyngeal movements were significantly altered during the swallowing function. However, only few studies have quantitatively evaluated the swallowing function after oral cancer surgery.

The purpose of this study was to quantitatively investigate the factors that affect swallowing dysfunction and to analyze the pathology of dysphagia in patients who underwent surgery for oral cancer.

\section{MATERIALS AND METHODS}

\section{Patient characteristics}

This study was approved by the Institutional Review Board of Kumamoto University Hospital (No.1359) and Kumamoto Health Science University (No.18004). After obtaining informed consent, all patients enrolled in this study. All patients who underwent oral cancer surgery from July 2007 to April 2015 at Kumamoto University Hospital and had swallowing dysfunction, were enrolled in this study.

\section{Food Intake LEVEL Scale}

The swallowing function assessment included the Food Intake LEVEL Scale (FILS) score at discharge. The FILS is an assessment method reported by Fujishima and his colleagues $[5,6]$ to evaluate the severity of dysphagia based on a 10-point scale. For this study, we collected information from the patients' medical records retrospectively and obtained their FILS scores at discharge or transfer. This scale measures the degree of dysphagia in patients by examining the extent of oral intake of food every daily. Levels 1 to 3 indicate varying degrees of nonoral intake of food. Levels 4 to 6 indicate varying degrees of oral food intake and alternative nutrition. Levels 7 to 10 imply varying degrees of oral food intake (Table 1).

\section{Videofluoroscopy examination}

Of the 70 patients who underwent oral cancer surgery

Table 1. Food intake LEVEL scale (FILS)

\section{No oral intake}

Level 1: No swallowing training is performed except for oral care.

Level 2: Swallowing training not using food is performed.

Level 3: Swallowing training using a small quantity of food is performed.

Oral intake and alternative nutrition

Level 4: Easy-to-swallow food less than the quantity of a meal (enjoyment level) is ingested orally.

Level 5: Easy-to-swallow food is orally ingested in one to two meals, but alternative nutrition is also given.

Level 6: The patient is supported primarily by ingestion of easy-to-swallow food in three meals, but alternative nutrition is used as a complement.

Oral intake alone

Level 7: Easy-to-swallow food is orally ingested in three meals. No alternative nutrition is given.

Level 8: The patient eats three meals by excluding food that is particularly difficult to swallow.

Level 9: There is no dietary restriction, and the patient ingests three meals orally, but medical considerations are given.

Level 10: There is no dietary restriction, and the patient ingests three meals orally (normal). 
from July 2007 to April 2015 in our institution, 23 underwent videofluoroscopic swallowing study (VFSS) examination for severe swallowing dysfunction after surgery. Videofluoroscopy (VF) examination for dysphagia was performed using an X-ray radiography equipment (Sonial Vision Safire II; Shimadzu Corporation, Kyoto, Japan). The examination was conducted in the lateral projection with the patient in the sitting position. A $120 \%$ barium sulfate suspension (Bali Top 120; Kaigen Pharma Co. Ltd.) or iopamiron (Iopamiron 370; Bayer AG, Leverkusen, Germany) was administered as a $3-5 \mathrm{~mm}$ bolus in a cup or syringe. We undertook the following steps to analyze the images: First, we recorded movies of the VF examination in the lateral view using a Mini DV. Second, we connected the digital video camera containing the Mini DV to a personal computer with IEEE 1394 cables. Next, data recorded by the Mini DV were converted into the Audio Video Interleave format with video editing software wherein the data comprised 30 frames per second. We then randomly selected 23 recoded data. Finally, we assessed the usefulness of the selected movies with a digitized video imaging software program and evaluated the swallowing function after the first swallowing action.

The average duration from oral cancer surgery to VF examination was 25.3 days (range, 6-134 days; median, 22.0 days). The VF image analysis was performed using the Ditect Image Processing Products (DIPP)-Motion V software (DITECT Corp., Tokyo, Japan). The 23.5-mm diameter coin of the calibration marker was positioned on the lateral neck. The five parameters were as follows.

Maximum hyoid bone movement means displacement of the hyoid bone between forward and upward which is quite essential during a healthy swallow in terms of airway protection and efficient bolus passage into the esophagus [7]. The movement would be limited with the degree of suprahyoid muscle resection for oral cancer surgery.

Deterioration of laryngeal sensation following radiation therapy sometimes leads to swallowing dysfunction with aspiration [8]. Miyaji et al. [9] defined a laryngeal elevation delay time (LEDT) to assess laryngeal elevation itself during swallowing. However, sometimes, the parameter is examined for assessing the degree of deterioration of laryngeal sensation presented as the delay of the laryngeal elevation due to radio therapy for oral cancer.

Leonard et al. [10] measured pharyngeal clearance in- directly using pharyngeal constriction ratio (PCR) examined with VFSS. We used PCR in the present study, based on the assumption that PCR would be enhanced with tumor resection at the base of tongue during oral cancer surgery.

The fourth parameter was residue in the vallecular and pear-shaped depression (pyriform sinuses). Residue in the vallecular is associated with tongue base movement, and residue in the pyriform sinuses is subjected with dysfunction of the anterior laryngeal movement and cricopharyngeal muscle [11].

The final parameter was Penetration-Aspiration Scale (PAS) [12] score. The PAS was used to evaluate aspiration and penetration.

The method of analyzing the VF video using DIPPMotion V is shown in Fig. 1. The y-axis is drawn from the anterior and superior tips of the third cervical vertebra to the anterior and inferior tips of the fifth cervical vertebra. The $\mathrm{x}$-axis is drawn orthogonal to the $\mathrm{y}$ axis. The measurement point of the hyoid bone was its anterior lower edge. Using the anterior and inferior tips of the fifth cervical vertebra as the origin, the maximum forward and upward movements of the hyoid bone during the swallowing reflex relative to its resting position were measured. The LEDT frame by frame playback of the 30 frames per second moving image of the side view was performed, and the time from when the tip of the

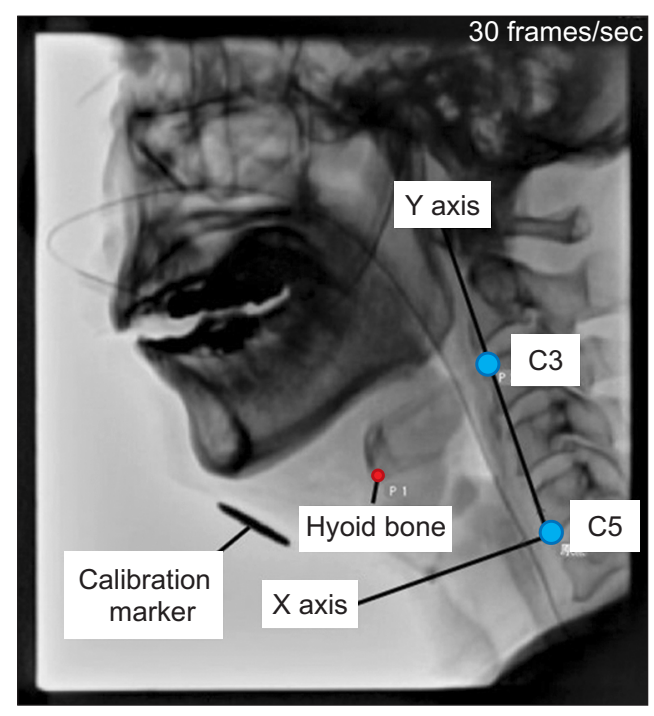

Fig. 1. Settings of $x$ and $y$ axes for video analysis. C3, third cervical vertebra; $\mathrm{C} 5$, fifth cervical vertebra. 

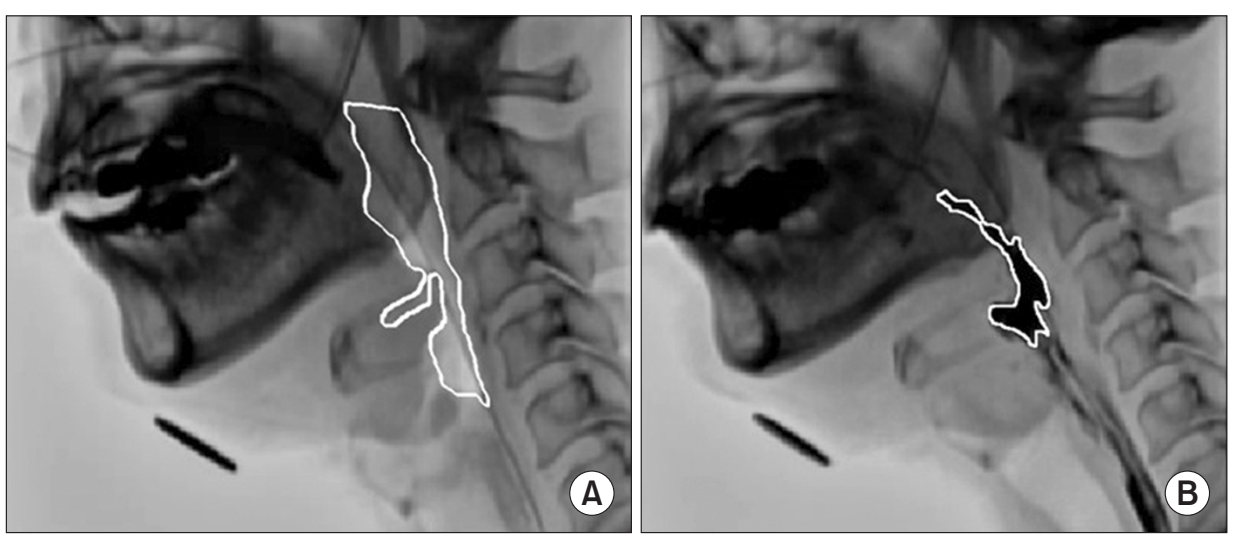

Fig. 2. Pharyngeal constriction ratio (PCR): (A) $\mathrm{PA}_{\text {hold }}$ (B) $\mathrm{PA}_{\max }$. $\mathrm{PCR}=\frac{\mathrm{PA}_{\max }}{\mathrm{PA}_{\text {hold }}}$.

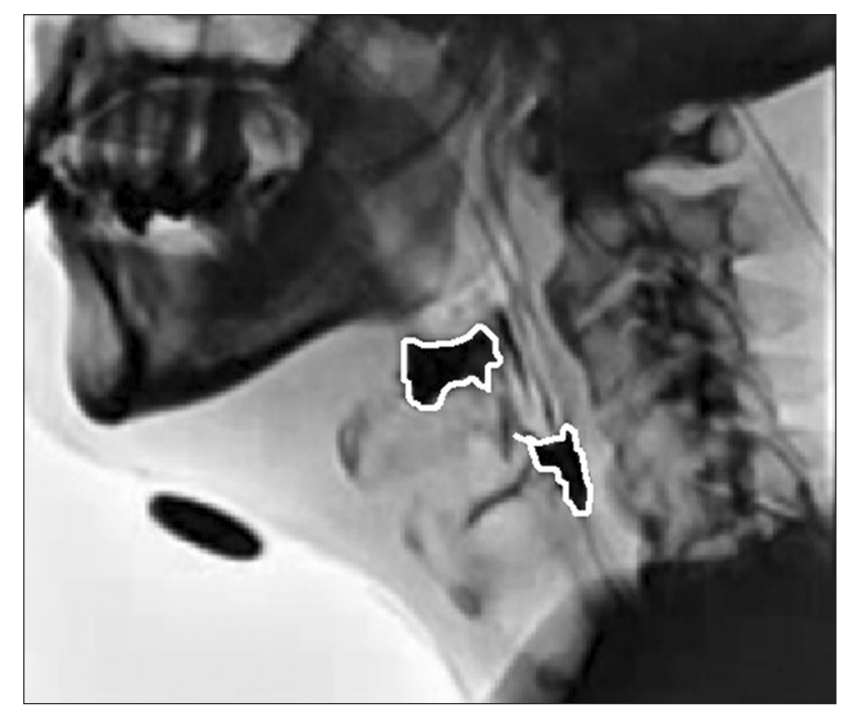

Fig. 3. Residues in the vallecular and pyriform sinuses. The areas marked with white lines were defined as the residues in the vallecular and pyriform sinuses.

contrast agent reached the bottom of the pear-shaped depression to when the laryngeal elevation reached the maximum position was measured. The PCR was presented as the $\mathrm{PA}_{\text {max }} / \mathrm{PA}_{\text {hold }}$ ratio. The $\mathrm{PA}_{\text {hold }}$ was measured in the lateral view during the hold position. The $\mathrm{PA}_{\max }$ was defined by the outline of the same structures during maximal constriction (Fig. 2). The residues in the vallecular and pyriform sinuses were measured by marking the area of the contrast agent remaining in each part of the vallecular and pyriform sinuses after swallowing (Fig. 3).

The recorded videos were rearranged randomly and evaluated by three speech therapists who had a minimum of 5 years' experience in the evaluation and training for dysphagia; the median value of the measurements by the three individuals was used for each evaluation item.

\section{Statistical analysis}

The intra-rater reliability of each parameter for VF examination was examined using the intra-rater correlation coefficient (the Spearman correlation) between each pair of data obtained on different days for a week. The interrater reliability of each parameter was examined using an inter-rater correlation coefficient (the Spearman correlation) between each pair of data evaluated by the three speech pathologists.

Multiple regression analysis as multivariate analysis was performed in the present study with the FILS score as the objective variable and factors demonstrating the characteristic for each case (age, tumor size, resection of the tongue base, suprahyoid muscle resection, segmental mandibulectomy, neck dissection and radiation therapy) as explanatory variables. We determined these using the actual size of a specimen removed in surgery. First, univariate analysis was performed between the FILS at discharge and age, tumor size, tongue base resection (none $=0$, unilateral $=1$, bilateral $=2$ ), suprahyoid muscle resection (none $=0$, unilateral $=1$, bilateral $=2$ ), segmental mandibulectomy (none $=0$, existence $=1$ ), neck dissection (none $=0$, unilateral $=1$, bilateral $=2$ ), and radiation therapy (none $=0$, existence=1) using Spearman rank correlation coefficient. Second, a factor with a high correlation coefficient was selected as the explanatory variable. Baseline variables with a p-value $<0.20$ in the univariate analysis were included using multiple regression analysis. Therefore, multiple regression analysis was performed using FILS as the objective variable and variables with a $p$-value $<0.20$ in the univariate analysis as the explanatory variables.

Additionally, multivariate analysis using multiple regres- 
Table 2. Clinical information of the 70 patients who underwent oral cancer surgeries

\begin{tabular}{lc}
\hline \multicolumn{1}{c}{ Variable } & Value \\
\hline Sex & \\
Male & 50 \\
\hline Female & 20 \\
\hline Age $(\mathrm{yr})$ & $64.6 \pm 12.2(21-85)$ \\
\hline Tumor size & 6 \\
\hline T1 & 43 \\
\hline T2 & 12 \\
\hline T3 & 9 \\
\hline T4 & \\
\hline Tumor location & 47 \\
\hline Tongue & 16 \\
\hline Floor of mouth & 5 \\
\hline Buccal mucosa & 2 \\
\hline Gingiva & \\
\hline Reconstruction procedure & 16 \\
\hline Free flap & 10 \\
\hline Forearm & 6 \\
\hline RAMC & 29 \\
\hline Pedicled flap & 22 \\
\hline PMMC & 3 \\
\hline LDMC & 4 \\
\hline PLM & 25 \\
\hline None & 18 \\
\hline Radiation therapy & 52 \\
\hline Received & \\
\hline None & \\
\hline
\end{tabular}

Values are presented as mean \pm standard deviation (range). RAMC, rectus abdominis musculocutaneous; PMMC, pectoralis major myocutaneous; LDMC, Latissimus dorsi musculocutaneus; PLM, platysma musculocutaneous.

sion analysis was performed between the maximum hyoid bone movement, LEDT, PCR, residue in the vallecular and pyriform sinuses, and PAS score as the objective variables and one of the main factors representing the characteristics of each case as the explanatory variables, and age was treated as an adjustment factor in VFSS. Moreover, statistical analysis in the present study was performed using JMP 14.2 (SAS Institute Inc., Cary, NC, USA).

\section{RESULTS}

Seventy patients who enrolled in this study underwent
Table 3. Clinical information of the 23 patients received videofluoroscopy examination

\begin{tabular}{|cc|}
\hline \multicolumn{1}{c}{ Variable } & Value \\
\hline Sex & 14 \\
\hline Male & 9 \\
\hline Female & $65.3 \pm 11.7(40-82)$ \\
\hline Age (yr) & \\
\hline Tumor size & 12 \\
\hline T1 & 5 \\
\hline T2 & 6 \\
\hline T3 & \\
\hline T4 & 12 \\
\hline Tumor location & 6 \\
\hline Tongue & 3 \\
\hline Floor of mouth & 2 \\
\hline Buccal mucosa & \\
\hline Gingiva & 7 \\
\hline Reconstruction procedure & 4 \\
\hline Free flap & 3 \\
\hline Forearm (FA) & 15 \\
\hline RAMC & 12 \\
\hline Pedicled flap & 1 \\
\hline PMMC & 2 \\
\hline LDMC & 1 \\
\hline PLM & \\
\hline None & 8 \\
\hline Radiation therapy & 15 \\
\hline Received & \\
\hline None & \\
\hline
\end{tabular}

Values are presented as mean \pm standard deviation (range). RAMC, rectus abdominis musculocutaneous; PMMC, pectoralis major myocutaneous; LDMC, Latissimus dorsi musculocutaneus; PLM, platysma musculocutaneous.

Table 4. Results of the multiple regression analysis $(n=70)$

\begin{tabular}{lccc}
\hline & $\beta$ & t & p-value \\
\hline Age & -0.297 & -2.57 & $*$ \\
Tumor size & -0.074 & -0.64 & 0.52 \\
Resection of the tongue base & -0.356 & -2.99 & $* *$ \\
Segmental mandibulectomy & -0.166 & -1.48 & 0.14
\end{tabular}

${ }^{*} \mathrm{p}<0.05,{ }^{* *} \mathrm{p}<0.01$.

oral cancer surgery. The subjects included 50 males and 20 females, with age ranging from 21 to 85 years. The primary tumor locations were the following: tongue in 47 patients, 
Table 5. Results of the multiple regression analysis in VF image analysis $(n=23)$

\begin{tabular}{|c|c|c|c|c|}
\hline & Experimental variables & $\beta$ & $\mathbf{t}$ & p-value \\
\hline \multirow[t]{6}{*}{ Forward movement of the hyoid bone } & Tumor size & $-0.664(0.411)$ & 3.57 & $* * *(0.0019)$ \\
\hline & Resection of the tongue base & $-0.135(-0.055)$ & -0.61 & $0.55(0.6588)$ \\
\hline & Segmental mandibulectomy & $-0.364(0.011)$ & -1.77 & $0.09(0.3449)$ \\
\hline & Suprahyoid muscle resection & $-0.285(0.071)$ & -1.31 & $0.20(0.1834)$ \\
\hline & Neck dissection & $-0.429(0.119)$ & -2.10 & $*(0.1080)$ \\
\hline & Radiation therapy & $-0.068(-0.069)$ & -0.068 & $0.75(0.7533)$ \\
\hline \multirow[t]{6}{*}{ Upward movement of the hyoid bone } & Tumor size & $-0.357(0.057)$ & -1.73 & $0.09(0.2136)$ \\
\hline & Resection of the tongue base & $-0.255(-0.013)$ & -1.17 & $0.25(0.4430)$ \\
\hline & Segmental mandibulectomy & $-0.300(0.015)$ & -1.42 & $0.17(0.3282)$ \\
\hline & Suprahyoid muscle resection & $-0.552(0.236)$ & -2.89 & $* *(0.0260)$ \\
\hline & Neck dissection & $-0.331(0.032)$ & -1.54 & $0.13(0.2780)$ \\
\hline & Radiation therapy & $-0.177(-0.048)$ & -0.81 & $0.42(0.6191)$ \\
\hline \multirow[t]{6}{*}{ LEDT } & Tumor size & $0.106(0.042)$ & 0.51 & $0.61(0.2508)$ \\
\hline & Resection of the tongue base & $0.391(0.192)$ & 2.01 & $0.06(0.0456)$ \\
\hline & Segmental mandibulectomy & $-0.053(0.039)$ & -0.26 & $0.80(0.2761)$ \\
\hline & Suprahyoid muscle resection & $0.392(0.191)$ & 2.00 & $0.06(0.0463)$ \\
\hline & Neck dissection & $0.225(0.083)$ & 1.08 & $0.29(0.1614)$ \\
\hline & Radiation therapy & $0.101(0.040)$ & 0.48 & $0.63(0.2539)$ \\
\hline \multirow[t]{6}{*}{ PCR } & Tumor size & $0.484(0.158)$ & 2.48 & $*(0.0684)$ \\
\hline & Resection of the tongue base & $0.675(0.383)$ & 3.96 & $* *(0.0030)$ \\
\hline & Segmental mandibulectomy & $0.204(-0.054)$ & 0.93 & $0.36(0.6524)$ \\
\hline & Suprahyoid muscle resection & $0.262(-0.027)$ & 1.18 & $0.25(0.5067)$ \\
\hline & Neck dissection & $0.302(-0.003)$ & 1.39 & $0.18(0.3990)$ \\
\hline & Radiation therapy & $0.235(-0.038)$ & 1.09 & $0.29(0.5629)$ \\
\hline \multirow[t]{6}{*}{ Residue in the vallecular } & Tumor size & $-0.041(-0.009)$ & -0.19 & $0.85(0.9558)$ \\
\hline & Resection of the tongue base & $0.435(0.103)$ & 2.11 & $*(0.1294)$ \\
\hline & Segmental mandibulectomy & $-0.098(-0.086)$ & -0.44 & $0.66(0.8822)$ \\
\hline & Suprahyoid muscle resection & $-0.216(-0.047)$ & -0.97 & $0.34(0.6156)$ \\
\hline & Neck dissection & $-0.175(-0.086)$ & -0.78 & $0.44(0.8822)$ \\
\hline & Radiation therapy & $0.347(0.036)$ & 1.66 & $0.11(0.2666)$ \\
\hline \multirow[t]{6}{*}{ Residue in the pyriform sinuses } & Tumor size & $0.318(0.017)$ & 1.51 & $0.14(0.3240)$ \\
\hline & Resection of the tongue base & $0.302(0.002)$ & 1.39 & $0.17(0.3764)$ \\
\hline & Segmental mandibulectomy & $-0.097(0.028)$ & 1.59 & $0.12(0.2881)$ \\
\hline & Suprahyoid muscle resection & $0.334(-0.084)$ & -0.43 & $0.67(0.8666)$ \\
\hline & Neck dissection & $-0.072(-0.088)$ & -0.32 & $0.75(0.9025)$ \\
\hline & Radiation therapy & $0.172(-0.061)$ & 0.79 & $0.44(0.7011)$ \\
\hline \multirow[t]{6}{*}{ PAS } & Tumor size & $0.094(-0.083)$ & 0.43 & $0.67(0.8585)$ \\
\hline & Resection of the tongue base & $0.038(-0.091)$ & -1.60 & $0.12(0.9267)$ \\
\hline & Segmental mandibulectomy & $0.129(-0.075)$ & 0.58 & $0.56(0.7946)$ \\
\hline & Suprahyoid muscle resection & $-0.223(-0.041)$ & -1.00 & $0.32(0.5767)$ \\
\hline & Neck dissection & $0.302(-0.087)$ & -0.31 & $0.75(0.8960)$ \\
\hline & Radiation therapy & $0.235(-0.038)$ & -0.14 & $0.88(0.5629)$ \\
\hline
\end{tabular}

The numbers in parentheses are the adjusted $\mathrm{R}^{2}$.

LEDT, laryngeal elevation delay time; PCR, pharyngeal constriction ratio; PAS, Penetration-Aspiration Scale. 
floor of the mouth in 16 patients, buccal mucosa in five patients, and gingiva in two patients. Forty-five patients underwent reconstruction of which 16 patients used free flap and 29 patients used pedicled flaps (Table 2).

Of the 70 patients, 23 underwent VFSS examination for severe swallowing dysfunction after surgery. The subjects included 14 males and 9 females, with age ranging from 40 to 82 years (Table 3). Among the 23 patients, the hyoid bone was lifted to the mandible in 10 cases during surgery. Additionally, oral phase disorders that affected piecemeal deglutition were observed in all the patients. Nine patients presented with aspiration or laryngeal penetration of which two patients demonstrated aspiration.

The intra-rater correlation coefficient of each parameter for the VF examination was 0.817-0.968 $(\mathrm{p}<0.0001)$. The inter-rater correlation coefficient of each parameter for the VF examination was $0.592-0.956$ ( $p=0.0055$ to $\mathrm{p}<0.0001)$.

\section{Factors affecting FILS}

Mean FILS value was 7.2. Baseline variables with a pvalue of $<0.20$ in the univariate analysis were age, tumor size, resection of the tongue base, and segmental mandibulectomy. Multiple regression analysis was performed using FILS as the objective variable and age, tumor size, resection of the tongue base and segmental mandibulectomy as the explanatory variables. The FILS presented statistically significant negative correlation with age (worse in older patients) and resection of the tongue base (worse in patients with extended resection). The remaining variables were not significant (Table 4).

\section{VF image analysis}

Although, the range of inter-rater correlation coefficients among the three raters for VF image analysis was somewhat wide (0.592-0.956), a significant correlation was observed, therefore, the median value was used as the value in the present study. The maximum forward movement of the hyoid bone was significantly influenced by the tumor size. The maximum upward movement of the hyoid bone was significantly influenced by the presence or absence of the suprahyoid muscle resection. PCR and residue in the vallecular were significantly influenced by the resection of the tongue base. The remaining variables were not significant (Table 5).

\section{DISCUSSION}

The degree of dysphagia subsequent to oral cancer surgery varies by resection range and position. Studies that have quantitatively evaluated the pathology revealed that some patients can start oral intake immediately while others can take time to ingest; however, the cause is not clearly understood. It is essential to explain to the oral cancer patients at the preoperative stage the degree of dysphagia and obtain their informed consent.

\section{Factors affecting FILS}

The prototype of the FILS, which is a 10-point observerrating scale to measure the severity of dysphagia, has been widely used since 1993 without any formal reliability and validity testing [5,6]. Kojima et al. [13] retrospectively evaluated potential prognostic factors for dysphagia in order to examine the feasibility of predicting the outcome using FILS. Wakabayashi et al. [14] assessed the prevalence of skeletal muscle mass loss using FILS and its association with swallowing function in patients with dysphagia after cardiovascular surgery. FILS is commonly used to evaluate the stage of dysphagia in patients. However, only few studies have evaluated swallowing function after oral cancer surgery using FILS.

Several previous studies have demonstrated that elderly people experience swallowing dysfunction caused by a delayed swallowing reflex and related muscle atrophy. Kaneko [15] showed that with advanced age, larynx prolapse tends to occur, which leads to a decline in swallowing function. Nishikubo et al. [16] quantitatively demonstrated through videofluorographic and manometric examinations that the swallowing reflex was delayed and upper esophageal sphincter (UES) opening was impaired by aging. Conversely, in a quantitative analysis of oral and oropharyngeal resection, Pauloski et al. [17] identified that the degree of tongue base resection is the most important determinant of postoperative swallowing function. Fujimoto et al. [1] indicated that tongue base resection of $>50 \%$ is not a significant factor for oral intake; however, it significantly affects the composition of the final diet. Resection of more than half of the tongue base causes swallowing dysfunction in both the oral stage and pharyngeal stage. Overall, these studies support our present data, which indicate that at discharge, patient age and size of the resected tongue base after oral 
cancer surgery are the two main parameters that determine postoperative swallowing ability when evaluated with FILS. However, radiotherapy of the laryngopharynx sometimes leads to functional disabilities including swallowing dysfunction $[8,18,19]$. Maruo et al. [8] showed that laryngeal sensation was significantly reduced from 1 to 3 month after (chemo)radiotherapy. In addition, severe impairments of swallowing function have been observed years after completion of treatment with conventional chemoradiation [18]. The swallowing dysfunctions are attributed to mucosal and muscular inflammation, fibrosis, reduced saliva secretion, and deterioration of laryngeal sensation [19]. In consideration with these previous studies, since we conducted a short follow-up period in the present study, the enhancement of the late chronic phase of the radiation therapy, in which the main side effect is a swallowing dysfunction, was not studied sufficiently at this time. In the future, we need to investigate the effect of radiation therapy on the swallowing function for a long period (e.g., more than 5-year post-radiation treatment).

\section{VF image analysis}

In our study, the maximum forward hyoid bone movement was significantly influenced by tumor size and neck dissection. The maximum upward hyoid bone movement was significantly influenced by the presence or absence of suprahyoid muscle resection. PCR was significantly influenced by tumor size and resection of the tongue base. Residue in the vallecular was significantly influenced by the resection of the tongue base.

Muscles involved in the hyoid bone movement include the geniohyoid muscle, mylohyoid muscle, stylohyoid muscle, and digastric muscle [20]. The morphology of the suprahyoid muscles suggests that based on structural properties, the geniohyoid has the most potential to displace the hyoid in the anterior direction, and the mylohyoid has the most potential to displace the hyoid in the superior direction [20]. This study suggested that the expansion of the resection range due to the large size of the tumors along with damage to both the geniohyoid and mylohyoid muscles may reduce hyoid bone movement, which can consequently induce severe postoperative swallowing dysfunction.

In addition, maximum forward hyoid bone movement was significantly influenced by neck dissection and tumor size. Ohashi et al. [21] reported that spatial analysis revealed significant decreases after neck dissection in the maximum distance of the UES opening, the maximum distance of hyoid bone movement in both the anterior and superior directions, and the maximum velocity the of hyoid bone movement. Neck scarring from neck dissection may affect the reduced hyoid bone movement.

It is clear that large tumor sizes increase the extent of resection. In this study, tumor size was influenced by the maximum forward hyoid bone movement and PCR. However, it is also important to not only consider the tumor size but also the reconstruction method. Fujimoto et al. [1] demonstrated that eating capabilities had been attained by patients reconstructed with free flaps rather than pedicled flaps. It is essential to explore other factors that would enhance the reconstruction procedure in the future.

Another important point is the pharyngeal swallowing pressure. Using manometry, Leonard et al. [10] demonstrated that there was a high inverse correlation between PCR (objective surrogate measure of pharyngeal strength) and the peak pharyngeal swallowing pressure. Pauloski et al. [22] demonstrated that the tongue base to posterior pharyngeal wall contact is essential but not sufficient for effective pharyngeal clearance. Based on these perspectives in the previous studies, we assume that sufficient duration of tongue base to posterior pharyngeal wall contact is critical to provide adequate pharyngeal bolus driving pressure.

In the present study, we demonstrated that the maximum hyoid bone movement, PCR, and residue in the vallecular are statistically correlated with factors such as tumor size, resection of the tongue base, suprahyoid muscle resection, and neck dissection, demonstrating the characteristics of each case. The result suggested that rehabilitative maneuvers that would improve the hyoid bone mobility and strengthen the pharyngeal constriction are quite beneficial after surgery. Based on the previous studies, widely accepted rehabilitative compensatory maneuvers to improve and strengthen the pharyngeal constriction are effortful swallow [23] or the Mendelsohn maneuver [24]. Effortful swallow requires patients to "swallow hard," which improves posterior tongue base movement and anterior movement of the pharyngeal wall [23]. Conversely, the Mendelsohn maneuver, or voluntary prolongation of the hyolaryngeal elevation at the peak of the swallow, has been used to treat patients with 
pharyngeal dysphagia for many years [24]. Additionally, the maneuver has been shown to increase UES opening duration and pharyngeal peak contraction [25], which would contribute to the impaired laryngeal elevation after oral cancer surgery.

\section{Limitations of this study}

First is the inconsistent bolus volume due to piecemeal deglutition caused by the resection of the tongue base, which is significantly enhanced on VF images. There are conflicting opinions on the positive or negative effect of bolus volume on the swallowing movement, especially considering the laryngeal elevation and the extent of pharyngo-esophageal segment (PES) opening increased by the bolus size $[26,27]$. Therefore, precise measurement of the bolus volume, which is lacking in the present study, would be critical for future prospective studies.

Second is that the patient sample is very heterogeneous, with very varied surgeries such as the resection of the tongue base, suprahyoid muscle resection, segmental mandibulectomy, and neck dissection, where the indication depends on the tumor extension. Therefore, such surgical procedure variables would have led to some limitations in the statistical analysis due to the heterogeneity of the samples. In the future prospective analysis, we need to unify the surgical procedure dependent on certain oral cancer tumor extensions.

Third is the evaluation period for the VF examination. In this study, the average duration from oral cancer surgery to VF examination was 25.3 days (range, 6-134 days; median, 22.0 days). Swallowing function after oral cancer surgeries may be altered over time by flap volume and swallowing rehabilitation interventions [28]. It is important to note that there is no evaluation in the long-term follow-up postoperatively. Moreover, lack of preoperative VFSS is surely a strong bottle neck to evaluate postoperative swallowing function. This rating would be necessary for future prospective studies.

The last limitation is the smaller number of patients in each subgroup for the statistical analysis. In the present study, we had seven factors as explanatory variables (age, tumor size, resection of the tongue base, suprahyoid muscle resection, segmental mandibulectomy, neck dissection and radiation therapy) representing the characteristics of each case. Therefore, for the multivariate analysis using multiple regression analysis, we need at least 70 cases (10 cases at least for each parameter) of VFSS as the minimum ideal number for the statistic, despite the actual insufficient number, which is 23 , in the present study. Therefore, multivariate analysis using multiple regression analysis was performed between the maximum hyoid bone movement, LEDT, PCR, residue in the vallecular and pyriform sinuses, and PAS score as the objective variables and one of the main factors representing the characteristics of each case as the explanatory variables, age treated as an adjustment factor in the VFSS. It is necessary to perform further analysis in a prospective manner with a higher number of patients.

\section{Conclusion}

At discharge, patient age and size of the resected tongue base $(>50 \%)$ after oral cancer surgery are the two main parameters that these factors can limit postoperative swallowing ability when evaluated with FILS. We demonstrated that maximum hyoid bone movement, PCR and residue in the vallecular are significantly correlated with factors demonstrating the characteristic for each case. These results suggested that effortful swallow or the Mendelsohn maneuver, which strengthens pharyngeal constriction, might be quite beneficial for patients following after oral cancer surgery. Further analysis, in a prospective manner, needs to be performed with an increased number of patients.

\section{CONFLICT OF INTEREST}

No potential conflict of interest relevant to this article was reported.

\section{ACKNOWLEDGMENTS}

This work was presented at the International Conference of the 40th Anniversary of AMS-KKU Foundation in Conjunction with the 4th Allied Health Sciences in Bangkok Thailand November 7, 2019. This work was supported by Kumamoto Health Science University special fellowship (No. 2018-D-01).

\section{AUTHOR CONTRIBUTION}

Conceptualization: Narihiro K. Methodology: Narihiro K. Formal analysis: Keigo M, Takumi M. Project admin- 
istration: Yorihisa O. Writing - original draft: Narihiro K. Writing - review and editing: Yoshihiko K, Yasuhiro S. Approval of final manuscript: all authors.

\section{REFERENCES}

1. Fujimoto Y, Hasegawa Y, Yamada H, Ando A, Nakashima T. Swallowing function following extensive resection of oral or oropharyngeal cancer with laryngeal suspension and cricopharyngeal myotomy. Laryngoscope 2007;117:1343-8.

2. Matsunaga $\mathrm{K}$, Mori $\mathrm{K}$, Asamura S, Nagata T, Isogai $\mathrm{N}$. Swallowing function in patients who underwent hemiglossectomy and reconstruction with a pectoralis major myocutaneous flap. Nihon Gekakei Rengo Gakkaishi 2008;33:829-36.

3. Pauloski BR, Rademaker AW, Logemann JA, Colangelo LA. Speech and swallowing in irradiated and nonirradiated postsurgical oral cancer patients. Otolaryngol Head Neck Surg 1998;118:616-24.

4. Pauloski BR, Logemann JA, Fox JC, Colangelo LA. Biomechanical analysis of the pharyngeal swallow in postsurgical patients with anterior tongue and floor of mouth resection and distal flap reconstruction. J Speech Hear Res 1995;38:110-23.

5. Fujishima I. [Textbook of rehabilitation for swallowing disorders associated with stroke]. 3rd ed. Tokyo, Japan: Ishiyaku Publishers; 1993. p. 143-52.

6. Kunieda K, Ohno T, Fujishima I, Hojo K, Morita T. Reliability and validity of a tool to measure the severity of dysphagia: the Food Intake LEVEL Scale. J Pain Symptom Manag 2013;46:201-6.

7. Cook IJ, Dodds WJ, Dantas RO, Kern MK, Massey BT, Shaker R, et al. Timing of videofluoroscopic, manometric events, and bolus transit during the oral and pharyngeal phases of swallowing. Dysphagia 1989;4:815.

8. Maruo T, Fujimoto Y, Ozawa K, Hiramatsu M, Suzuki A, Nishio N, et al. Laryngeal sensation and pharyngeal delay time after (chemo)radiotherapy. Eur Arch Otorhinolaryngol 2014;271:2299-304.

9. Miyaji H, Umezaki T, Adachi K, Sawatsubashi M, Kiyohara H, Inoguchi T, et al. Videofluoroscopic assessment of pharyngeal stage delay reflects pathophysiology after brain infarction. Laryngoscope 2012;122:2793-9.
10. Leonard R, Belafsky PC, Rees CJ. Relationship between fluoroscopic and manometric measures of pharyngeal constriction: the pharyngeal constriction ratio. Ann Otol Rhinol Laryngol 2006;115:897-901.

11. Logemann JA. Evaluation and treatment of swallowing disorders. Austin, TX: Pro-ED; 1998. p. 71-133.

12. Rosenbek JC, Robbins JA, Roecker EB, Coyle JL, Wood JL. A penetration-aspiration scale. Dysphagia 1996;11:93-8.

13. Kojima A, Imoto Y, Osawa Y, Fujieda S. Predictor of rehabilitation outcome for dysphagia. Auris Nasus Larynx 2014;41:294-8.

14. Wakabayashi H, Takahashi R, Watanabe N, Oritsu H, Shimizu Y. Prevalence of skeletal muscle mass loss and its association with swallowing function after cardiovascular surgery. Nutrition 2017;38:70-3.

15. Kaneko I. [A cinefluorographic study of hyoid bone movement during deglutition]. Nihon Jibiinkoka Gakkai Kaiho 1992;95:974-87.

16. Nishikubo K, Mise K, Ameya M, Hirose K, Kobayashi T, Hyodo M. Quantitative evaluation of age-related alteration of swallowing function: videofluoroscopic and manometric studies. Auris Nasus Larynx 2015;42:1348.

17. Pauloski BR, Rademaker AW, Logemann JA, McConnel FM, Heiser MA, Cardinale S, et al. Surgical variables affecting swallowing in patients treated for oral/ oropharyngeal cancer. Head Neck 2004;26:625-36.

18. Chang YC, Chen SY, Lui LT, Wang TG, Wang TC, Hsiao TY, et al. Dysphagia in patients with nasopharyngeal cancer after radiation therapy: a videofluoroscopic swallowing study. Dysphagia 2003;18:135-43.

19. Jensen K, Lambertsen K, Grau C. Late swallowing dysfunction and dysphagia after radiotherapy for pharynx cancer: frequency, intensity and correlation with dose and volume parameters. Radiother Oncol 2007;85:7482.

20. Pearson WG Jr, Langmore SE, Zumwalt AC. Evaluating the structural properties of suprahyoid muscles and their potential for moving the hyoid. Dysphagia 2011;26:345-51.

21. Ohashi N, Iwai T, Tohara H, Chiba Y, Oguri S, Koizumi $\mathrm{T}$, et al. Swallowing function in advanced tongue cancer patients before and after bilateral neck dissection following superselective intra-arterial chemoradiotherapy for organ preservation: a case-control study. 
Oral Radiol 2019;35:230-8.

22. Pauloski BR, Logemann JA. Impact of tongue base and posterior pharyngeal wall biomechanics on pharyngeal clearance in irradiated postsurgical oral and oropharyngeal cancer patients. Head Neck 2000;22:12031.

23. Hoffman MR, Mielens JD, Ciucci MR, Jones CA, Jiang JJ, McCulloch TM. High-resolution manometry of pharyngeal swallow pressure events associated with effortful swallow and the Mendelsohn maneuver. Dysphagia 2012;27:418-26.

24. Mendelsohn MS, Martin RE. Airway protection during breath-holding. Ann Otol Rhinol Laryngol 1993;102:941-4.

25. Boden K, Hallgren A, Witt Hedstrom H. Effects of three different swallow maneuvers analyzed by videomanometry. Acta Radiol 2006;47:628-33.

26. Leonard RJ, Kendall KA, McKenzie S, Goncalves MI, Walker A. Structural displacements in normal swallowing: a videofluoroscopic study. Dysphagia 2000;15:146-52.

27. Wintzen AR, Badrising UA, Roos RA, Vielvoye J, Liauw L. Influence of bolus volume on hyoid movements in normal individuals and patients with Parkinson's disease. Can J Neurol Sci 1994;21:57-9.

28. Ohkubo M, Sugiyama T, Ohira M, Yamamoto M, Iguchi T, Shibahara T, et al. Swallowing rehabilitation affects period of hospitalization after surgery for tongue cancer. Bull Tokyo Dent Coll 2017;58:19-26. 